Comments received included 'Great service' 'Kept in touchkeeps your mind on it'

'Found the health psychology input helpful. Having someone to talk to who was interested in me cheered me up" Really useful to have someone to report back to in terms of achieving weight loss goals "Extremely helpful in terms of explaining why weight loss was so important'

Think it is really helped having someone who is supportive and willing to listen is a big help. You don't feel you're achieving anything if you've got nobody to bounce it off'.

'I feel more confident that I can make changes'

'First Class Service -, I feel a lot more positive'

'The support I received at the NAFLD clinic was useful, it was good to talk about weight and why it was important to change things. Made me realise I needed to start making changes for myself and my family. We have all lost weight'

'Felt supported from the team. Telephone support really worked for me. Made me feel good and realise the progress I had made'

Conclusions The results have identified that patients who attended the multi-disciplinary clinic made substantial improvements to their lifestyle. Average daily step count increased over the 6 month period, from 1635 to 5958. 82\% patients reported an increase in activity levels; $27 \%$ patients had been shielding but reported doing home based exercise programmes. Average self-reported weight loss at 6 months was $5.5 \mathrm{~kg}$ (range $0.5 \mathrm{~kg}-23 \mathrm{~kg}$ ). There were also a reduction in the average anxiety and depression scores using HADS, from 7.4 to 6 for anxiety and 6.3 to 3.3 for depression.

The majority of patients, who engaged reported an increase in self-efficacy in terms of achieving weight loss. An important outcome (measured via patient's qualitative feedback) was around self-confidence and recognition that small changes were a great step in the right direction.

A learning point when we are able to fully resume normal service delivery is to try and increase the number of patients benefitting from this service. We see scope to potentially access patients earlier in primary care before they are referred to the Gastroenterology Team thus reducing waiting times and freeing up secondary care medical staff for more complex cases.

Although the Liver Nurse Specialist team were fully on board with identifying suitable patients, not all eligible patients took the service up. We are not sure the reason for this, although we are aware that the cohort of patients with NAFLD are often considered the 'hard to reach' patients, faced with multiple challenges, which may mean 'health' can slip down their priority list. It is worth noting that the majority of patients seen had complex long standing issues contributing to their poor health. Aspects of family functioning including conflict and divorce; emotional eating, insomnia, depression, anxiety were highlighted and in most cases were worked with throughout the support sessions from the psychologist.

Overall, it is considered that this new pathway and multidisciplinary clinic has been very worthwhile. There have been noticeable improvements identified through the use of appropriate assessment tools and from positive qualitative patient and clinician feedback. In terms of future plans funding has been secured to work with innovation colleagues to provide a virtual version of supported self-management for less complex patients. This will allow a greater through put of patients and help further implement the new NAFLD pathway and multidisciplinary approach.

\section{PTH-60 IMPACT OF SARS-COV-2 PANDEMIC ON THE IBD ADVICE LINE - A SINGLE CENTRE EXPERIENCE}

Uma Selvarajah* ${ }^{*}$ Catarina Martins, Bridgette Fraser, Uma Selvarajah, Neerav Joshi. Chelsea And Westminster Hospital NHS Foundation Trust, Harrow, UK

\subsection{6/gutjnl-2021-BSG.375}

SARS-CoV-2 pandemic has significantly affected delivery of gastroenterology services across UK. Transition to telephone clinics, redeployment of clinical staff and reduction in endoscopy capacity have resulted in additional pressures on the IBD advice line. We retrospectively analysed Chelsea and Westminster Hospital NHS Foundation trust's IBD advice line to evaluate the impact of the pandemic and to identify areas to improve upon.

Method Phone calls to IBD advice line are recorded on an excel sheet by the IBD nurses; this was used to identify list of patients who called the advice line between January - February 2021. Electronic operating system, Cerner, was used to mine data relevant to each phone call. Outcome parameters audited include patient demographics, reasons for call, outcomes from call, faecal calprotectin levels in documented flares, time since last clinic appointment and time to next outpatient appointment. This project was locally registered with the hospital's clinical audit team.

Results 209 patients contacted the service between 4/1/2021 to $04 / 02 / 2021$. We were able to interrogate notes for 199 patients. 55\% $(n=109)$ of patients had Crohn's disease, 39\% had ulcerative colitis and $6 \%$ had indeterminate colitis. 55\% of patients were female $(n=109)$ and median age was 32 years (youngest $=18$, oldest $=72) .90 \%(n=179)$ of the phone calls were from patients and remaining phone calls were from GPs and allied health care professionals. 10\% of patients needed more than one attempt to call them back. $26.6 \%(n=53)$ of the phone calls were related to flares, $13.6 \%(n=27)$ of the phone calls were directly related to COVID-19 and $13.6 \%(n=27)$ were related to further guidance on treatment. $22.6 \%$ of the phone calls needed IBD sisters to chase investigations, liaise with secretaries to chase appointments and convey results of investigations.

Phone calls directly related to COVID-19 include advice regarding shielding, implications for medications during periods of acute illness and advice regarding vaccinations.

Of the 53 patients who had documented flare, 27 had a raised faecal calprotectin of $>250 \mathrm{ug} / \mathrm{g}$. 28.1\% $(\mathrm{n}=56)$ phone calls resulted in telephone advice from IBD nurses and 14.1\% $(n=28)$ needed escalation to a gastroenterology consultant or team.

$26 \%(n=51)$ of the patients were spoken to in an outpatient telephone clinic with in the last 4 weeks and $31 \%(n=$ 61) patients had an appointment coming up in the next 4 weeks.

Discussion SARS-CoV-2 pandemic has put additional pressures on the IBD advice line. In January 2021, there were $20 \%$ more phone calls than January 2020 at our centre. It is reassuring that there is no gender predominance in who accesses the advice line. Younger median age is consistent with peak incident age but with aging population and increasing prevalence of IBD in this cohort, more need to be done to ensure that there are no barriers to older population accessing the helpline. As intended, significant proportion of the phone calls were related to disease flares, however IBD nurses were only able to offer advice to $28.1 \%$ of the patients. This highlights 
the need for strong adhoc clinician support to deal with complex patients and dedicated time for ongoing professional development for the IBD nurses. High volume of phone calls resulting in chasing investigations, conveying results and liaising with administrative team on behalf of patients necessitate better education about the IBD advice line. $57 \%$ of the patients who called had recently had a clinic appointment or was due one; with very little training given on running telephone clinics, one wonders about the clinical effectiveness of telephone clinics and whether the clinicians are able to address all of patients' concerns during these appointments.

During the COVID-19 pandemic, IBD advice line has been a valuable resource to patients. From safety and cost effectiveness (IBD advice line cost - $£ 39 /$ call, A\&E attendance - $£ 148$ / visit) point of view, it continues to enhance patient experience.

\section{PTH-61 FAECAL ELASTASE MAY HAVE A ROLE IN THE INVESTIGATION OF IRON DEFICIENCY ANAEMIA}

Faisal Shaikh*, Sadaf Dar, Richard Shenderey, Susanna West, Lionel Gracey-Whitman, Ahmad Zakaria, Fahad Shaikh, Chris Healey, David Clements. Airedale Hospitals NHS Trust, Keighley, UK

\subsection{6/gutjnl-2021-BSG.376}

Introduction Faecal elastase-1 (Fel-1) is often used for investigation of diarrhoea and if exocrine pancreatic insufficiency (EPI) is suspected ${ }^{1}$. It is common in routine clinical practice that patients with EPI have iron deficiency anaemia (IDA). Although logical that EPI patients are prone to IDA due to malabsorption, ${ }^{2,3}$ the association has not yet been shown in the published literature ${ }^{4}$. We wanted to see how strong the positive association might be between low Fel-1 (and therefore EPI) and IDA.

Methods Patients with low Fel-1 $<200 \mathrm{mcg} / \mathrm{g}$ were identified retrospectively from December 2019 to November 2020. Endoscopy, radiology, coeliac serology and ferritin results were reviewed. None of the low Fel-1 patients had an identified cancer or any other cause for IDA. Statistical analysis was performed to study the association of IDA in patients with low Fel-1.

Results 301 Fel-1 results were available over one year period 47/301 (16\%) patients were identified with low Fel-1

$26 / 47$ were females

Mean age $66(\mathrm{SD} \pm 15)$

7/47 (15\%) low Fel-1 patients had IDA and unremarkable investigations

7/7 (100\%) with low Fel-1 and IDA had diabetes or impaired glucose tolerance

$7 / 40$ (17\%) with low Fel-1 and normal ferritin had diabetes

26/254 (10\%) with normal Fel-1 had IDA

228/254 (90\%) with normal Fel-1 had normal iron

Sensitivity of finding IDA in low Fel-1 patients 0.15 (95\% CI $0.06-0.28)$

Specificity $0.90(95 \%$ CI $0.85-0.93)$

Positive predictive value $0.21(95 \%$ CI $0.09-0.39)$

Negative predictive value 0.90 (95\%CI $0.80-0.89$ )

Positive likelihood ratio 1.5 (95\%CI $0.67-3.20)$

Conclusions (1) Our study shows that low Fel-1 is positively associated with IDA.

(2) Fel-1 may have a role in investigation of unexplained iron deficiency anaemia.
(3) Fel-1 is useful in the IDA pathway especially in patients who also have diabetes

(4) A low Fel-1 may help with identifying EPI as a cause of IDA and reduce repeat cycles of endoscopic investigations by treating EPI.

\section{REFERENCES}

1. Lam KW, Leeds J. How to manage: patient with a low faecal elastase Frontline Gastroenterology 2021;12:67-73.

2. Shintakuya R, Uemura K, Murakami $Y$, et al. Sarcopenia is closely associated with pancreatic exocrine insufficiency in patients with pancreatic disease. Pancreatology. 2017 Jan-Feb;17(1):70-75. doi: 10.1016/j.pan.2016.10.005. Epub 2016 Oct 11. PMID: 27743711

3. De la Iglesia-García D, Huang W, Szatmary P, et al. Efficacy of pancreatic enzyme replacement therapy in chronic pancreatitis: systematic review and meta-analysis. Gut 2017:66:1354-1355.

4. Dominguez-Munoz, Juan Enrique, Castineira, Margarita, Larino-Noia, Jose, et al. (2010). M1391 Iron Metabolism and Iron-Deficiency Anemia in Patients with Chronic Pancreatitis. Gastroenterology. 138. 10.1016/S0016-5085(10)61815-X

\section{PTH-62 THE SAFETY AND QUALITY OF ULTRASOUND-GUIDED LIVER BIOPSY IN CLINICAL PRACTICE}

Luca Cardillo*, Momtaj Khalik, Nivian Sukirthan, Hazem Rizk, Rebecca Greenhalgh, Laith Alrubaiy. London North West University Healthcare NHS Trust, London, UK

\subsection{6/gutjnl-2021-BSG.377}

Introduction Liver biopsy constitutes an invaluable diagnostic, prognostic, decision-making and research tool in patients with liver disease. Multiple approaches and techniques are described, however evidence is scanty in many aspects. Audits evaluating complications of liver biopsies, use of different biopsy needles, biopsy size and communication between the clinician and pathologist are paramount to ensuring high-quality care. This study assesses local liver biopsy services in a non-specialised liver unit against guidelines from the British Society of Gastroenterology, the Royal College of Radiologists and the Royal College of Pathology. Findings provide a paradigm for audit and quality improvement of local liver biopsy services in other hospitals.

Methods This is a retrospective audit of electronic records. 110 ultrasound-guided percutaneous liver biopsies performed at our trust in 2019 were examined. Patients undergoing biopsy at tertiary centres were excluded. Information regarding consent, anticoagulation/antiplatelet medications, clotting and platelet levels, biopsy needles used, biopsy sample size, complications, and the pathology report were collected and anonymised. Data was processed in Microsoft Excel.

Results The current clinical practice was assessed against published guidelines. Informed consent was obtained from all patients. However, this was documented in procedural notes only in $88 \%$ of occasions. A minority of patients $(7 \%)$ were on anticoagulation/antiplatelet therapy; this was correctly withheld on $99 \%$ of procedures. INR prior to biopsy was $>1.4$ in only $1 \%$ of patients; this was adequately corrected. Platelets were checked in all patients and did not require correction. Targeted biopsies from a focal lesion correctly utilised an 18 $G$ needle in most instances. Non-targeted biopsies for diffuse parenchymal disease (DPD), however, did not utilise the recommended needle size of 16 gauge in $77 \%$ of biopsies. Indeed, DPD biopsies provided sufficient sample sizes $(>20$ $\mathrm{mm})$ in $41.2 \%$ of cases. Complications were reported in $4 \%$ of patients, and were mostly minor. Pathology reports answered the clinical question in $91 \%$ of cases, and were received within 10 days of biopsy in $83 \%$. 
Correction: PTH-60 Impact of SARS-CoV-2 pandemic on the IBD advice line- a single centre experience

Selvarajah U, Martins C, Fraser B, et al. PTH-60 Impact of SARS-CoV-2 pandemic on the IBD advice line- a single centre experience. Gut 2021;70:A203-A204. doi:10.1136/ gutjnl-2021-BSG.375

Nur Nurmahomed should be included in the abstract as first author.

(C) Author(s) (or their employer(s)) 2022. No commercial re-use. See rights and permissions. Published by BMJ.

Gut 2022;71:e7. doi:10.1136/gutjnl-2021-BSG.375corr1

(A) Check for updates 